\title{
Integrating Usability and Accessibility in Information Assurance Education
}

\author{
Azene Zenebe, Claude Turner \\ Bowie State University
}

\begin{abstract}
One of the emerging challenges in information assurance (IA) is usability and accessibility. Usability refers to the extent in which a security system is easy to learn, remember and use by users; and accessibility refers to the quality of a security system being in situation to be used by all kind of users including those who have specific types of cognitive, physical, or perceptual disabilities. The majority of computer and information security faculty who teach security and assurance courses are not familiar with the topics of usability and accessibility, especially the latter, and do not incorporate these topics into their classes despite that a number of results of research on usability are presented in SOUPS and other HCI conferences. What is lacking is strong effort to integrate these resources into teaching and research. Hence, this project attempts to narrow the gap by building and supporting a national community of faculty members who will integrate usability and accessibility in Information Assurance (IA) education and research.
\end{abstract}

\section{INTRODUCTION}

One of the emerging challenges in information assurance (IA) is usability and accessibility. Usability refers to the extent in which a security system is easy to learn, remember and use by users; and accessibility refers to the quality of a security system being in situation to be used by all kind of users including those who have specific types of cognitive, physical, or perceptual disabilities.

Underlying security controls and measures are complex and hence threats can arise from human errors and cognitive limitations during the installation, configuration, use and maintenance of these security systems. In his article, Schneier stated: "to the average home user, security is an intractable problem. ... there are still a dizzying array of rules, options, and choices that users have to make. How should they configure their anti-virus programs? What sort of backup regime should they employ? What are the best settings for their wireless network?"[6], pp. 243).

A large number of existing security techniques and tools are developed without considering the actual needs and capabilities of the user[4]. Hence, they are not usable. Since the widely adopted visual CAPTCHA [1] is inaccessible for millions of users who are visually impaired (Holman, Lazar, Feng and D'Arcy, 2007; Cranor and Garfinkel, 2005), audio CAPTCHAs are introduced in some web sites to these groups of users. To address security concerns, the audio CAPTCHAs adopt background noise and dictation from different speakers, making it difficult and timeconsuming to use [2].

The majority of computer and information security faculty who teach security courses are not familiar with the topics of usability and accessibility, especially the latter, and do not incorporate these topics into their classes despite that a number of results of research on usability are presented in SOUPS and other HCI conferences and a few literature also exist. What is lacking is strong effort to integrate these resources into teaching and

\author{
Jinjuan Feng, Jonathan Lazar, Mike O'Leary \\ Towson University
}

research. Hence, this project attempts to narrow the gap by building and supporting a national community of faculty members who will integrate usability and accessibility in Information Assurance (IA) education and research.

\section{METHODS}

We propose to build and continuously support a national community of faculty members to integrate usability and accessibility in Information Assurance (IA) education by developing lecture materials, providing workshops and conducting collaborative research. Some of the other major activities include:

- Develop a set of learning and teaching materials that allow instructors to effectively integrate usability and accessibility concepts and strategies in IA courses,

- Develop an online web portal through which faculty and community members can access the educational materials, communicate with each other, and share knowledge and experiences,

- Disseminate the educational materials and the online portal through conferences and seminars,

- Develop faculty who can teach usability, accessibility, and IA courses and conduct research in the related fields, and

- Develop a research mentorship program, where security, usability, and accessibility researchers in Towson University and Bowie State University invite community college and junior faculty to work jointly with them on research projects.

\section{RESULTS}

The project is at its early stage. However, teaching and learning materials have been developed, research has started, and a workshop was delivered in May 2009. The teaching modules that have been developed include:

Usability and Computer and Information Security System: Using this lecture material, the readers will come to understand the concept of usability, computer and information security and their interconnection. First, definitions are presented. Second, the needs for and challenges associated with usable security systems are presented. Finally, framework for studying usability of computer and information security systems are presented and discussed.

Human capability and IA: This material highlights the fundamentals of human cognitive, perceptual, and physical capabilities and their implications for the design and development of security mechanisms. For example, the design of any access control mechanism that requires user login needs to consider the human memory and information processing capacity. Although strong passwords that are long and consist of a mixture of 
numbers, letters (both uppercase and lowercase), and special symbols seem to be desirable from the security perspective, they may exceed the capacity of human memory limit. In that case, the users will, instead of remembering the password, adopt alternative solutions that violate the fundamental security rule that passwords should only be stored in users' brain. Understanding this fundamental human capability is important for IA professionals.

Requirement analysis for usable IA applications: A security mechanism is not successful unless it fully accommodates the needs of the intended users as well as the organization. Besides the traditional technical requirements, requirement analysis needs to cover a diversified range of areas including usability requirements, reliability requirements, user requirements, and environmental requirements. This resource introduces the commonly used requirement analysis methods such as observation, survey, interview, focus group, and cognitive walkthrough as well as presents how they can be used in IA.

Population diversity and IA: In order to design and develop accessible IA applications, IA professionals need to understand the dramatic diversity of the human population and realize that the design not only need to work for the 'typical users', but also those with capabilities towards either ends of the spectrum. This lecture material will address critical topics regarding the diversity of human cognitive, physical, perceptual capabilities, the capability range for the 'typical user group', as well as the underrepresented populations in the design world, such as the elder users and the users with impairments.

With respect to faculty development, a one-day workshop on usability and accessibility was conducted. Twenty-two participants, mostly faculty, attended the workshop led by two experts in the fields of usability and accessibility. The goal of the usability presentation was to explore usability, human cognitive processes and capabilities, and their relationship. These cognitive capabilities are foundational to usability of human-computer interfaces that can function efficiently and effectively in assisting users their tasks as well as in protecting their information and computer resources from security breaches such as from phishing, virus, worm and Trojans attacks.

The accessibility presentation provided a background on web accessibility, laws that require accessibility, and interface guidelines that help explain how to make a web site accessible. The presentation examined various web-based security features, discussing how these features can cause accessibility problems. The accessible design alternatives were also discussed.

With respect to research, multiple projects are underway including one that focuses on making CAPTCHAs accessible for visually impaired individuals $[3,5]$.

\section{CONCLUSION AND FUTURE WORKS}

The project aims at reaching a large body of faculty in Computer Science (CS), Information Systems (IS), and Management Information Systems (MIS) fields who are teaching students with diversified career goals. Different from local training programs that only benefit a small audience, the virtual community we aim to build will have the potential to connect faculty who are involved in IA education nationwide. Introducing the critical concepts of usability and accessibility into IA courses will make an impact on IA education. A large number of students in the CS, IS, and MIS fields will be equipped with the knowledge of usable and accessible security techniques and apply those knowledge into practice. This will greatly promote the development of IA techniques and tools that are user-friendly, universally accessible, and easily adoptable.

Future activities will include development of teaching materials on usability guidelines for design, development and implementation of usable IA applications, Usability evaluation methods for IA applications and mechanisms, Usable

Maintenance Mechanism for IA systems, IA policy and usability; and designing IA applications for individuals with different types of impairments; conducting a local seminar and a conference in the summer of 2010; undertaking collaborative research; and disseminating the developed teaching materials and the online web portal both nationally and locally.

\section{ACKNOWLEDGMENTS}

This work is supported by NSF grant DUE 0830865.

\section{REFERENCES}

[1] Ahn, L.V., Blum, M. and Langford, J. Telling humans and computers apart automatically. Communications of ACM, 47 (2), 56-60.

[2] Bigham, J.P. and Cavender, A.C. Evaluating existing audio CAPTCHAs and an interface optimized for non-visual use. In Proceedings of the 27th international conference on Human factors in computing systems, ACM, Boston, MA, USA, 2009, 1829-1838.

[3] Holman, J., Lazar, J., Feng, J.H. and D'Arcy, J. Developing usable CAPTCHAs for blind users. In Proceedings of the 9th international ACM SIGACCESS conference on Computers and accessibility, ACM, Tempe, Arizona, USA, 2007, 245246.

[4] Sasse, A. and Flechais, I. Usable Security. In Cranor, L.F. and Garfinkel, S., Eds. Security and Usability: Designing Secure Systems That People Can, O'Reilly Media, California, 2005.

[5] Sauer, G., Holman, J., Lazar, J., Hochheiser, H., and Feng, J. (2009, in press). Accessible Privacy and Security: A universally Usable Human-Interaction Proof. Paper accepted for special issue of Universal Access in the Information Society Journal.

[6] Schneier, B. Schneier on security. Wiley Publishing, Inc., Indianapolis, 2008.

Authors Contact Information:

Azene Zenebe, Department of Management Information Systems, Bowie State University, azenebe@bowiestate.edu ; Claude Turner, Department of Computer Science, Bowie State University, ctuner@bowiestate.edu ; Jinjuan Feng, and Jonathan Lazar, Department of Computer and Information Sciences, Towson University; Mike O'Leary, Center for Applied Information Technology, Towson University 